\title{
CONOCIMIENTOS Y CONCEPCIONES ESTADÍSTICAS DE LOS ESTUDIANTES DE SECUNDARIA EN BRASIL
}

\author{
Cassio Cristiano Giordano \\ ccgiordano@gmail.com \\ https://orcid.org/0000-0002-2017-1195 \\ Faculdades Integradas de Guarulhos (FG-SP) \\ São Paulo, Brasil
}

Recibido: 17 de septiembre de 2020 Aceptado: 21 de mayo de 2021

\begin{abstract}
Resumen
Realizamos una investigación cualitativa, buscamos identificar los conocimientos y concepciones estadísticos de 86 estudiantes del último año de secundaria de una escuela pública brasileña, con edades comprendidas entre los 16 y 19 años, movilizadas en la resolución de problemas en el aprendizaje por proyectos. Adoptamos, en nuestro marco teórico, Análisis Exploratorio de Datos (AED) y Teoría de Concepciones. En nuestros procedimientos metodológicos utilizamos los resultados del Análisis Estadístico Implicativo (ASI), elaborados mediante el software CHIC (Clasificación Jerárquica Implicativa y Cohesiva), para evaluar el nivel de conocimiento de los estudiantes, mientras que empleamos el modelo ck $\varnothing$ en el análisis de sus concepciones. Al final de nuestras investigaciones, identificamos once concepciones movilizadas por estos estudiantes.

Palabras clave: Educación Estadística; Teoría de Concepciones; Análisis Estadístico Implicativo; Proyectos.

\section{STATISTICAL KNOWLEDGE AND CONCEPTIONS OF BRAZILIAN HIGH SCHOOL STUDENTS}

\begin{abstract}
We conducted a qualitative research, in which we sought to identify the knowledge and statistical concepts of 86 students in the last year of high school in a Brazilian public school, aged sixteen to nineteen years old, mobilized to solve problems in project-based learning. We adopted, in our theoretical framework, Exploratory Data Analysis (EDA) and Theory of Conceptions. In our methodological procedures, we make use of the Statistical Implicative Analysis constructs, elaborated using the software CHIC (Implicative and Cohesive Hierarchical Classification), in order to assess the student's knowledge level, while we employ the ck $\phi$ model in the analysis of their conceptions. At the end of our investigations, we identified eleven conceptions mobilized by these students.

Keywords: Statistical Education; Theory of Conceptions; Implicative Statistical Analysis; Projects.
\end{abstract}




\title{
CONHECIMENTOS E CONCEPÇÕES ESTATÍSTICAS DE ALUNOS DO ENSINO MÉDIO NO BRASIL
}

\begin{abstract}
Resumo
Realizamos uma pesquisa qualitativa, na qual buscamos identificar o conhecimento e as concepções estatísticas de 86 alunos do último ano do ensino médio de uma escola pública brasileira, com idades entre 16 e 19 anos, mobilizados na resolução de problemas na aprendizagem por meio de projetos. Adotamos, em nosso referencial teórico, a Análise Exploratória de Dados (AED) e a Teoria das Concepções. Em nossos procedimentos metodológicos utilizamos os resultados da Análise Estatística Implicativa (ASI), mediada pelo uso do software CHIC (Classificação Hierárquica Implicativa e Coesitiva), para avaliar o nível de conhecimento dos alunos, enquanto utilizamos o modelo ck $\not$ na análise de suas concepções. Ao final de nossas investigações, identificamos onze concepções mobilizadas por esses alunos. Palavras-chave: Educação Estatística; Teoria das Concepções; Análise Estatística Implicativa; Projetos.
\end{abstract}

\section{Introducción}

Este artículo encontramos algunos resultados finales de la tesis doctoral de Giordano (2020). Investigaciones recientes de miembros de Grupo de Trabajo de Educación Estadística de la Sociedad Brasileña de Educación Matemática (GT12 - SBEM) indican que los estudiantes de secundaria, aunque tienen algún conocimiento de estadística descriptiva, demuestran dificultades para relacionar sus conceptos básicos con los problemas diarios que enfrentan en su vida personal, escolar y profesional. Sin embargo, al participar activamente en el desarrollo de investigaciones estadísticas, tienden a cambiar su visión de esta ciencia, conforme lo que indican los resultados observados por Mendonça (2008), Conti (2009), Santana (2011), Quirós (2012), Dangió (2014), Albani (2015), Freitas (2016), Giordano (2016), Barberino (2016), Melo (2017), Cobello (2018), Oliveira (2019), entre otros.

La comprensión de la estadística es fundamental para la formación académica, la vida profesional y, sobre todo, para el pleno ejercicio de la ciudadanía en el siglo XXI. En este sentido, a partir de una extensa revisión de la literatura, lo consideramos como un elemento de amplio potencial para el desarrollo de la alfabetización estadística, en la perspectiva de Gal (2019). Este entendimiento está en línea con la Base Curricular Común Nacional - BNCC (Brasil, 2018) recientemente aprobada. La propuesta curricular vigente en aquella escuela (São Paulo, 2012, 2014) preveía la enseñanza de la estadística descriptiva (la enseñanza de la estadística inferencial no estaba incluida en el currículo) solo en el segundo semestre del tercer y último año de secundaria. Así, evaluamos sus conocimientos previos, a través del Análisis 
Estadístico Implicativo (ASI), antes de abordar la estadística a través de proyectos. Después de la finalización de la investigación de los estudiantes y su exposición a la comunidad escolar, buscamos identificar las concepciones movilizadas con respecto al contenido curricular esperado: variabilidad, medidas de tendencia central, medidas de dispersión y registros de representación (gráficos estadísticos y tablas de distribución de frecuencias - TDF). La pregunta que orientó nuestra investigación fue: "¿Qué concepciones los estudiantes de secundaria aprenden en la resolución de problemas relacionados con la estadística descriptiva, después del desarrollo de proyectos en esta área?".

En la próxima sección, presentamos más detalles sobre la naturaleza del problema investigado.

\section{Problema de investigación}

Desde la aprobación de la Base Curricular Común Nacional - BNCC (Brasil, 2018), los currículos de las redes educativas públicas y privadas brasileñas han tenido cambios profundos. Este documento normativo, elaborado por el Ministerio de Educación de Brasil - MEC, amplió el alcance de la Estadística y Probabilidad en la Educación Básica, creando una unidad temática específica para esta área, a ser enseñada para todos los años de Educación Primaria - niños y adolescentes con edades de seis a diecisiete años - con énfasis en el enfoque a través de proyectos. Las cinco unidades temáticas son: Números, Álgebra, Geometría, Cantidades y Medidas, Probabilidad y Estadística. Bajo la nueva visión de las metodologías activas, muy valoradas en el siglo XXI, el enfoque centenario, ahora llamado por muchos Aprendizaje Basado en Proyectos - ABP, gana un nuevo impulso.

Como aporte teórico para delinear nuestras investigaciones, la opción por Análisis Exploratorio de Datos - AED nos parece una opción natural, una vez que su abordaje de la Estadística valora la postura investigativa crítica por parte del estudiante y presupone una propuesta didáctico-pedagógica centrada en la investigación, por el profesor. Como características básicas de la AED, Batanero, Estepa y Godino (1991) destacan la posibilidad de generar situaciones de aprendizaje sobre temas de interés de los estudiantes, a partir de representaciones gráficas que favorezcan la percepción de variabilidad, la valorización de medidas de orden, que disminuyen posibles casos atípicos, el uso de diferentes escalas, además 
de la falta de necesidad de una teoría matemática compleja, con herramientas innecesarias en ese momento.

Gal (2019) destaca como conocimientos estadísticos básicos para que los docentes desarrollen trabajos basados en AED: reconocer la necesidad de manipular datos, saber producirlos, presentar familiaridad con los términos e ideas más elementales en Estadística, así como sus registros de representación tabular y gráfica, dominar nociones de probabilidad y conocer métodos de elaboración de análisis estadístico inferencial. Según el autor, el conocimiento estadístico, a desarrollar por los estudiantes, será el resultado de sus habilidades en cuanto al conocimiento estadístico, al conocimiento matemático, al conocimiento del contexto y del mundo y su capacidad para elaborar preguntas sobre el conocimiento, asociado a elementos de disposición, que implica su postura crítica, así como sus creencias y actitudes.

Costa (2012) enfatiza la necesidad de promover este tipo de investigaciones, en particular, con el trabajo a través de proyectos, es de suma importancia que el docente valore los conocimientos previos de los estudiantes, ya que, de esta manera, ellos asumen el protagonismo en la investigación. Porciúncula y Samá (2015) piensan en la opción de trabajar con proyectos de aprendizaje no solo como una metodología, sino como una forma de reflexionar sobre el rol en la sociedad. Para Batanero y Díaz (2004) los proyectos estadísticos motivan a los estudiantes, en detrimento de la resolución de ejercicios fuera de contexto. Para las autoras, la estadística es la ciencia de los datos, y estos no son solo números, sino números en contexto. Según Batanero y Díaz (2004), al trabajar con proyectos, el énfasis está en tareas que deben ser realistas.

Batanero y Díaz (2011), enfatizan que el desarrollo de proyectos de trabajo, orientados a la Educación Estadística, contribuye a la adquisición de las siguientes habilidades, fundamentales para el estudiante de secundaria: competencia lingüística comunicativa, competencia matemática, competencia de reconocimiento e interacción con el mundo físico, competencia para el tratamiento de la información y competencia digital, competencia social y ejercicio de la ciudadanía, competencia para "aprender a aprender", cuestionar, identificar y manejar las diversas técnicas y estrategias para enfrentar una misma situación problemática, competencia para logro de la autonomía e iniciativa personal.

En nuestra investigación buscamos identificar los cambios en las concepciones estadísticas de estudiantes durante la realización de la investigación estadística. Así, 
consideramos oportuno adoptar, en nuestro marco teórico, de la Teoría de las Concepciones. Según Balacheff y Gaudin (2002), el conocimiento no se puede reducir totalmente a comportamientos, pero tampoco se puede enseñar en su ausencia. Toda acción moviliza una cantidad considerable de conocimientos. Para desarrollar nuevos conocimientos, así como profundizar en los anteriores, es necesario movilizar concepciones, directamente relacionados con los problemas que enfrentan los estudiantes. Balacheff (2002) afirma que una concepción no puede ni debe separarse del contexto en el que surge el problema, que lo resalta y le da sentido.

Almouloud (2007) nos recuerda que las concepciones permiten interpretaciones, predicciones y construcción de modelos y, sobre todo, describen una parte de la estructura cognitiva, en nuestro caso, del estudiante. En nuestra investigación adoptaremos las definiciones de conocimiento y concepción de la Teoría de las Concepciones del modelo propuesto por Balacheff(2002). Para él, una concepción es una estructura mental, característica de un sujeto (en nuestro caso, el estudiante), constituida por un observador de su comportamiento (en nuestro caso, el investigador). El aprendizaje, a su vez, consiste en pasar de una concepción a una concepción nueva, más compleja e integral. Pretendemos, con nuestra investigación, identificar concepciones significativas y, si es posible, posibles cambios en las mismas.

En la siguiente sección, presentamos más detalles sobre la naturaleza del problema investigado.

\section{Procedimientos metodológicos}

Investigamos las concepciones movilizadas por los estudiantes en la resolución de problemas estadísticos, abordados a través de proyectos, antes y después de su realización, desde la planificación y toma de datos hasta el análisis final y divulgación de resultados. Optamos por el enfoque metodológico de la investigación cualitativa, en la perspectiva de Creswell (2010). Para evaluar el nivel de conocimientos de los estudiantes, antes del desarrollo de los proyectos, se aplicó un cuestionario con 29 preguntas estadísticas básicas, analizando las respuestas a través del Análisis Estadístico Implicativo (ASI). 
Los sujetos de investigación fueron 86 estudiantes del último año de secundaria de una escuela pública brasileña, con edades entre los dieciséis y diecinueve años. En junio de 2018, respondieron un cuestionario compuesto por 29 preguntas estadísticas, analizadas con la ayuda del software CHIC (Classification Hiérarchique Implicative et Cohesitive). Este software permite extraer información de un conjunto de datos, asuntos y atributos cruzados, reglas de asociación entre variables, indicando el índice de calidad de la asociación y representando una estructuración de estas variables, según lo establecido por Couturier y Gras (2005) y Gras et al. (2013). Prado (2002, p. 14) considera fundamental el uso de este software, ya que es capaz de revelar elementos significativos sobre las reflexiones de docentes y estudiantes sobre temas relacionados con el aprendizaje y la práctica pedagógica. Según ella, el software CHIC: "... ayudó a identificar relaciones inesperadas, lo que reveló nuevos conocimientos sobre el proceso de aprendizaje".

En la segunda etapa de la investigación, cuatro grupos de estudiantes (dos parejas y dos tríos), en noviembre de 2018, después del desarrollo del proyecto, respondieron tres preguntas sobre estadística descriptiva durante un período de una a tres sesiones de 100 minutos cada una, en tres días distintos de la misma semana. Estas respuestas, registradas a través de la producción escrita y grabaciones de audio de las interacciones entre los estudiantes del grupo, se analizan de acuerdo con la Teoría de las Concepciones.

En la siguiente sección, presentamos el marco teórico y metodológico que guió nuestras investigaciones.

\section{Marco teórico-metodológico}

El AED nos proporciona un extenso repertorio de métodos para un estudio detallado de los datos antes de adaptarlos. Tukey (1970) sugirió el uso de las siguientes medidas de resumen representativas de una muestra dada: los valores máximo y mínimo, la mediana y los cuartiles. Él consideraba que la mediana y los cuartiles eran funciones de la distribución empírica más robustas en comparación con otras medidas de resumen tradicionales, como la media y la desviación estándar. Estas medidas de resumen se utilizan en la organización y presentación de datos, enfatizando una comprensión más efectiva de la información subyacente al trabajo de investigación de censo o por muestreo. Según Coutinho y Miguel (2007, p. 1), esta propuesta 
se destaca en el contexto de transición “... de una perspectiva técnica, en el aspecto matemático es lo predominante y los modelos están preestablecidos, para un analítico, en la que se busca construir modelos a partir del estudio de datos observados". AED es más accesible, motivadora y creativa. Impregnado del espíritu investigativo que debe caracterizar la producción de conocimiento científico. Batanero (2001) señala que hasta entonces el análisis de datos se basaba principalmente en el cálculo estadístico, llevando a dos consecuencias: disminución de la importancia visual de la representación de los datos y la creencia de que para llegar a conclusiones sobre los datos era necesario recurrir necesariamente a inferencia, como modelo confirmatorio. Según la autora, los datos se componen de dos partes intrínsecamente relacionadas: "regularidad" o tendencia, y "desviaciones" o variabilidad.

Hasta la aparición de AED, el foco de los estudios estadísticos se basaba en la búsqueda de un modelo capaz de representar fielmente la regularidad de las observaciones. AED, no intenta imponer modelos iniciales, sino a partir de los datos para crearlos. Como características básicas de este enfoque, Batanero et al. (1991) destacan la posibilidad de crear situaciones de aprendizaje sobre temas de interés para los estudiantes, apoyándose en representaciones gráficas que favorezcan la percepción de variabilidades, la valoración de medidas de orden que minimizan posibles casos atípicos-, el uso de diferentes escalas, además de la falta de necesidad de una teoría matemática compleja, con herramientas innecesarias en ese momento. Batanero (2001) afirma que, para elegir una asignatura a abordar en las clases de Estadística, el docente debe tener en cuenta su potencial para generar situaciones de aprendizaje relacionadas con temas de interés del estudiante, el fuerte apoyo en representaciones visuales, valorando el uso de múltiples representaciones de datos, especialmente gráficos, priorizando la discusión entre estudiantes, la argumentación científica, en detrimento del uso de una teoría matemática compleja. Para Batanero et al. (1994), la estadística ha recibido tradicionalmente menos atención en los planes de estudios escolares que otros campos de las matemáticas. Los autores también añaden que gran parte de la investigación en esta área se llevó a cabo en situaciones experimentales en vez de explorar situaciones escolares, y aquellas realizadas en contextos escolares enfocarán mayoritariamente a la educación superior. Al realizar una investigación estadística en el ámbito escolar en la educación básica, tratamos de dar respuesta a esta demanda. 
El AED se ha extendido como filosofía de aplicación de la Estadística, gracias a la rápida popularización de las computadoras y al desarrollo de software estadístico accesible, con amplias posibilidades para el procesamiento de datos y para sus representaciones gráficas, según Batanero (1999). Para la autora, simplificó la vida de docentes y estudiantes, permitiéndoles acercarse a otras materias escolares, posibilitando el trabajo en equipo y posibilitando el desarrollo de proyectos interdisciplinares. Así, consideramos oportuno desarrollar nuestro enfoque a través de proyectos en esta perspectiva.

Para identificar los conocimientos previos de los estudiantes sujetos de la investigación, recorremos a ASI. Adoptamos la ASI en nuestra investigación como herramienta en el tratamiento de los datos porque nos permite visualizar, organizar, construir modelos representativos, así como analizar los fenómenos asociados a ellos (Gras; Almouloud, 2002). El desarrollo de ASI fue impulsado desde 1970 por los trabajos realizados y guiados por Régis Gras - profesor emérito de la Escuela Politécnica de la Universidad de Nantes -, al paso que su operacionalización se pasó en la de 1990, con la creación y mejora del auxilio del software CHIC - Clasificación Jerárquica Implicativa y Cohesiva, utilizada para el análisis de datos multivariados.

Originalmente criado por Gras, el software fue desarrollado para la computadora por dos de sus estudiantes: Almouloud y Harrisson Ratsimba-Rajohn. Actualmente, CHIC ha sido actualizado y mejorado por Raphaël Couturier. En nuestras investigaciones, usamos la versión 7.0 de 2014. La importancia de CHIC para ASI es tan grande que Gras (2015, p. 9) afirma que, sin CHIC, ASI sería simplemente un objeto matemático cultural especulativo, desprovisto de aplicaciones porque los cálculos serían intratables; sin ASI, la herramienta informática para procesar cálculos y gráficos, CHIC no podría dar sentido a los resultados numéricos y las representaciones que produce, los dejaría empobrecidos e inaccesibles para el control y la crítica. La ASI es un método de análisis de datos no simétrico que permite, a partir de un conjunto de datos que cruza una población de sujetos u objetos con un conjunto de variables, la extracción y estructuración del conocimiento en forma de reglas y regulaciones generalizadas $\mathrm{y}$, a partir de la contingencia de estas reglas, la explicación y, en consecuencia, una cierta previsión en las distintas ramas del conocimiento humano. Su origen es el modelado estadístico de cuasi-implicación: cuando la variable, o la conjunción de las variables, "X" es observada en la población, generalmente la variable "Y" también lo es (Orús; Zamora; Gregori, 2009). 
Almouloud, Gras y Régnier (2014) afirman que el Análisis Estadístico Implicativo es un campo teórico cuyo objeto matemático es el concepto de cuasi-implicación, distinguiéndose de la implicación lógica de la Matemática y la Lógica misma. ASI busca conocimiento, invariantes y atributos de las variables estudiadas. El método estadístico implicativo ha sido ampliamente utilizado en estudios cualitativos de reglas de asociación, mostrando grandes aportes a la investigación en Educación Matemática, permitiendo mostrar la dinámica de las conductas de los estudiantes en situaciones de resolución de problemas. Al contrario de lo que es legítimo en Matemáticas, donde todas las reglas generalmente no sufren excepción y el determinismo está siempre presente en las Ciencias Humanas y Sociales, las excepciones no solo están casi siempre presentes, como también deseadas (Orús et al., 2009). Según estos autores, deferentemente de los métodos de análisis simétrico basado en la distancia en la correlación, los conjuntos de reglas obtenidos pueden conducir a la hipótesis de causalidad. Dichos conjuntos se estructuran según diferentes características comunes complementarias (grafo implicativo, jerarquía orientada), estableciendo conceptos de intensidad, del envolvimiento, cohesión de clase, niveles significativos, etc. Según Couturier, Bodin y Gras (2004), el software CHIC permite la extracción de un conjunto de datos, cruzando sujetos y variables (o atributos), y reglas de asociación entre variables, proporcionando índices de calidad de las asociaciones y representando la estructuración de las variables obtenidas a través de tales reglas. Los indicadores con los que trabajamos en nuestra investigación fueron insertados en este software como variables binarias, asumiendo los valores 0 o 1 , representando, respectivamente, la ausencia y la presencia de la variable para cada individuo encuestado.

Utilizamos ASI para sondear los conocimientos previos de los estudiantes sujetos a nuestra investigación. Hasta entonces, estos estudiantes casi no tenían contacto con Estadística en ambiente escolar. Fue de nuestro interés evaluar lo que ya sabían sobre Estadística, antes de iniciar su aprendizaje en un enfoque a través de proyectos. El software CHIC nos permitió desarrollar el análisis implicativo, el análisis de similitud y el análisis cohesivo.

El foco de nuestra investigación radica en las concepciones. Fuertemente influenciada por la Teoría de las Situaciones Didácticas - TSD y por la Teoría de los Campos Conceptuales, según Oliveira y Coutinho (2011), la Teoría de las Concepciones tiene como objetivo estudiar las relaciones entre conceptos, conocimientos y concepciones que, los estudiantes pueden presentar acerca de cierto contenido matemático. Para Balacheff (1995), rescatar la noción de 
concepción es movilizar acciones en el sujeto, mediante la realización de una actividad. Esto, en situaciones específicas, puede actuar de manera racional y coherente para resolver el problema. Una concepción, en el modelo ckф, es un estado de equilibrio de un sistema, sujetomedio, considerando sus limitaciones e imposiciones, es decir, todo aquello que influya o interfiera en su operación. La concepción pertenece al sujeto y, por tanto, puede ser o no correcta desde el punto de vista del conocimiento de referencia. Otro aspecto importante de este modelo es que la concepción suele ser local en el sentido de trabajar para resolver un problema específico y no otro que apunte a un dominio de validez. Una concepción implica un cuádruple $(\mathrm{P}, \mathrm{R}, \mathrm{L}, \Sigma)$ :

- P es un conjunto de problemas en que $\varnothing$ es operacional;

- R es un conjunto de operadores (herramientas cognitivas para acción);

- L es un sistema de representación, que permite expresar los elementos de P y R;

- $\Sigma$ es una estructura de control, que garante la no contradicción de $\not$. En este cuádruple, un sujeto que se enfrenta a un problema por resolver puede tener varias concepciones sobre un mismo objeto matemático y movilizar una u otra, según el problema propuesto. Una concepción está compuesto por un conjunto de conocimientos y lo conocimientos, a su vez, está compuesto por un conjunto de concepciones, como vemos representado en la siguiente figura:

Figura 1. Relación entre concepciones, conocimiento y conceptos.

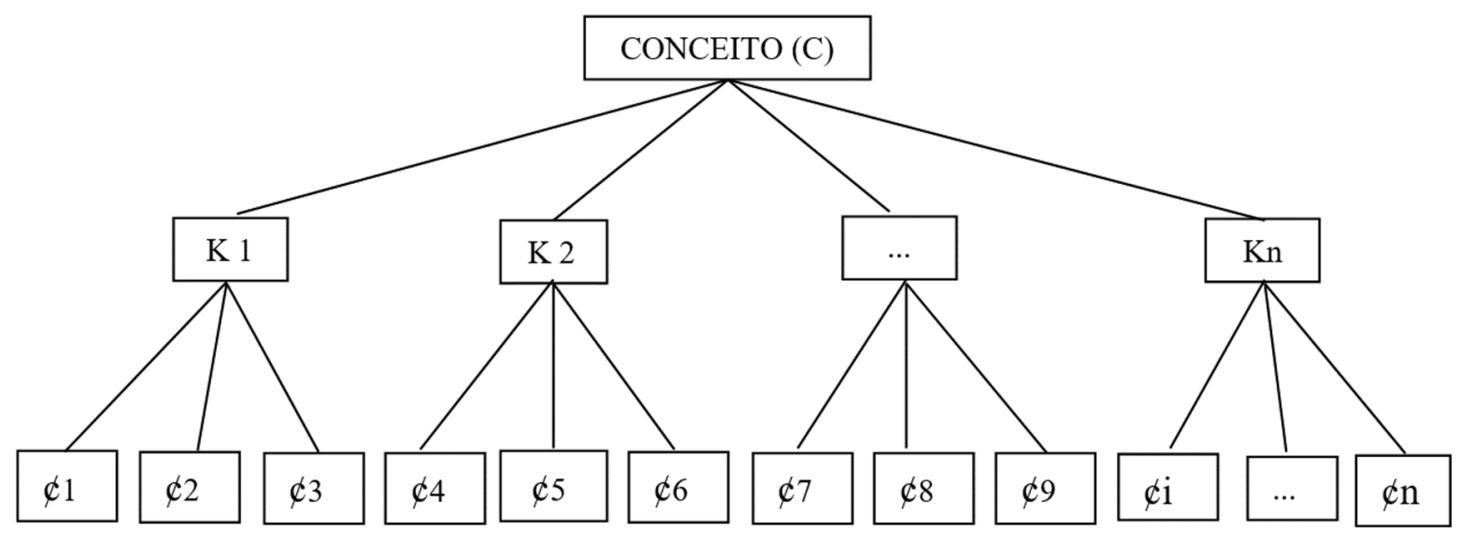

Fuente: Oliveira (2010, p. 43).

En la Figura $1, ф 1, ф 2, ф 3, \ldots$ e $ф$ n constituyen el conjunto de concepciones que forma lo de conocimientos, $\mathrm{K} 1, \mathrm{~K} 2, \ldots, \mathrm{Kn}$, que, a su vez, forma el concepto $\mathrm{C}$.

Como ya dijimos, en nuestra investigación, los estudiantes fueron capaces de movilizar ciertas concepciones al tratar de resolver problemas estadísticos similares a los que enfrentaron en el desarrollo de sus proyectos. Se espera que movilicen concepciones al realizar su propia 
investigación, lo que será útil para resolver los problemas. Las investigaciones de estos estudiantes involucran los más variados contextos, en situaciones a las que no están acostumbrados a enfrentar en las clases de matemáticas, debido a cuestiones curriculares ya presentadas por nosotros. Cada problema despierta en el estudiante comportamientos que son indicadores de conocimiento, mostrando evidencia de estrategias desarrolladas por él en la búsqueda de soluciones. Este conocimiento no puede atribuirse solo al estudiante, como una propiedad que le será intrínseca, ni solo al medio, ya que es una propiedad del sujeto en interacción con un sistema antagónico, con el medio. Tal interacción sólo será significativa si logra satisfacer las limitaciones que condicionan la viabilidad de la relación sujeto-medio. La resolución de problemas — resultado de una alteración más o menos severa en el equilibrio de esta relación -, es decir, el reequilibrio en esa relación, es la fuente y el criterio del saber. Así, el conocimiento se caracteriza por un estado de equilibrio dinámico en la interacción acciónretroacción, entre un sujeto $\mathrm{S}$ y un medio específico $\mathrm{M}$, según las limitaciones que impone la situación, que determinan o no su viabilidad.

El sistema didáctico está compuesto por el agente didáctico, humano o artificial, por el estudiante y el entorno. El conocimiento involucrado en su funcionamiento es una propiedad inherente del sistema estudiante-medio bajo las restricciones sostenidas por el agente didáctico, quien actúa como representante de la institución productora del sistema didáctico. El saber es un atributo del conocimiento, cuyo valor se reconstruye a partir del juicio del agente didáctico.

En sus intentos por resolver un determinado problema, el estudiante puede enfrentarse a varios obstáculos, que no implican una falta total de conocimientos, sino conocimientos movilizados fuera de su dominio de validez. Este obstáculo puede resistir a las contradicciones y continuar manifestándose incluso después de que el individuo se dé cuenta de su inexactitud y no solo está vinculado a factores estrictamente cognitivos, sino también a factores emocionales. Además, tiene que ver con el sentimiento de confianza que trae este “conocimiento" desplazado, que posiblemente sirvió en otras ocasiones para resolver muchos problemas. Por tanto, le corresponde al investigador identificar estos "errores" resistentes y demostrar que se organizan en torno a concepciones. El error, en este marco teórico, se entiende como un signo de una perturbación, el resultado de la diferencia reconocida por el sujeto entre el resultado esperado de una acción y la retroalimentación del entorno. El estudiante es capaz de reconocer esta diferencia como inaceptable, reacciona ante ella y busca un reequilibrio que 
le lleva a un nivel superior de conocimiento. Para Balacheff (1995), una concepción errónea requiere la noción de una conclusión aparentemente exitosa de una diversidad de problemas en los que el ciclo de formular el problema (expectativa), resolución del problema (acción) y reconstrucción del problema (revisión), se logra efectivamente. Tal concepción errónea tiene su propio campo de validez, ya que, como ya hemos señalado, el error no es solo el efecto de la ignorancia, la incertidumbre y la mala suerte que encontramos en las teorías empiristas del aprendizaje o behavioristas del aprendizaje, sino la consecuencia de conocimientos previos.

Lo que esencialmente distingue el paradigma de las concepciones erróneas del paradigma del error es el significado epistemológico de cada uno. Para Balacheff (1995), el estatus del conocimiento es diferente, ya que las concepciones erróneas implican la existencia de un conocimiento de referencia global y legítimo, mientras que el paradigma del error, particularmente en el problema de los obstáculos epistemológicos, solo requiere lo que explícitamente relaciona dos conocimientos desde una perspectiva de desarrollo. Así, el conocimiento es efímero y provisorio, pudiendo ser el objeto de cambios y ampliación de su campo de validez después de perturbaciones. El error persistente y el conocimiento contradictorio son objetos de análisis en la siguiente sección. Fischbein (1982) mostró que, en un mismo sujeto, la coexistencia de conocimientos contradictorios puede estar presente en aspectos tan fundamentales como los medios de validación. Este fenómeno parece, a primera vista, estar en contradicción con la idea de una construcción del conocimiento por adaptación que sugiere no contradicción. Vadcard (2000) agrega que la contradicción eliminaría este carácter operativo. El modelo de concepción, al asociar cada manifestación de conocimiento a una esfera práctica, permite explicar la contradicción que existe a los ojos del observador: la no coherencia, atribuida por una disyunción de las esferas de prácticas. Por tanto, este modelo permite preservar el carácter racional del conocimiento operacional, a la vez que proporciona una explicación de la coexistencia de estados contradictorios en un mismo sujeto.

Amparado por esos marcos teórico-metodológicos, presentaremos, en la próxima sección, nuestro análisis de resultados.

\section{Resultados y análisis}


Para comprender mejor esta sección, usted debe leer los Apéndices I y II, que se encuentran al final de este artículo. En primer lugar, analizamos la actividad realizada por 86 estudiantes, para identificar sus conocimientos estadísticos previos. En segundo lugar, analizamos la actividad realizada por cuatro grupos de estudiantes (dos parejas y dos tríos) para identificar concepciones movilizadas.

El cuestionario (Anexo I) fue aplicado en el salón de clase, en tres sesiones de 100 minutos cada una. La primera parte del cuestionario, consistía en una pregunta abierta y diez preguntas objetivas, se buscó identificar las concepciones y conocimientos de los estudiantes sobre estadística que contemplan el modelo de alfabetización estadística propuesto por Gal (2019). La segunda parte del cuestionario, compuesta por trece preguntas objetivas, intentó identificar el conocimiento estadístico y las evidencias de alfabetización probabilística de los estudiantes. En el desarrollo de la investigación, optamos por centrarnos en la estadística, dejando este análisis de alfabetización probabilística para futuros artículos. Finalmente, la tercera parte trae cinco preguntas objetivas, extraídas de las evaluaciones del Examen Nacional de Secundaria (ENEM) de los últimos años, (examen que permite la admisión, beca y / o financiamiento de estudios en la educación superior brasileña), nos permitió investigar el conocimiento estadístico de los estudiantes en relación con los contenidos específicos de estadística, previstos en el Currículo del Estado de São Paulo (São Paulo, 2012). Cabe mencionar que estos estudiantes no cursaron Estadística en la Secundaria de esta escuela, pues, según este documento, solo debe ser estudiado en un bimestre del tercer año.

Con la ayuda del software CHIC, realizamos el análisis de los gráficos implícitos, cohesivos y de similitud. Sin embargo, por razones de limitación de espacio, en este artículo presentaremos únicamente el gráfico implicativo, relacionado con la primera fase de la investigación: relevamiento de los conocimientos previos de los estudiantes sobre estadística.

En la segunda fase, presentaremos algunas concepciones movilizadas por ellos en la solución de problemas estadísticos. Construimos nuestro análisis con base en relaciones emergentes, desde el análisis implícito hasta la teoría clásica y la distribución binomial. Un análisis implicativo asocia las variables A y B de acuerdo con una meta-regla en "si esto sucede, probablemente aquello sucederá". Con cuadrados de colores, indicamos el nivel de confianza, para que podamos leer las respectivas asociaciones. En verde, tenemos valores entre 0,81 y 0,85; en azul, una variación entre 0,86 y 0,90 y, finalmente, en rojo, de 0,91 a 0,95. Esta escala 
está representada en la Figura 2. Los resultados del análisis implicativo, junto con los resultados del análisis de similitud y el análisis cohesivo, permitieron desarrollar un escenario para evaluar el conocimiento estadístico de los estudiantes.

En la fase experimental de nuestra investigación, construyeron sus conocimientos y desarrollaron su alfabetización estadística basándose en la resolución de problemas, luego del desarrollo de proyectos:

Figura 2. Gráfico Implicativo.
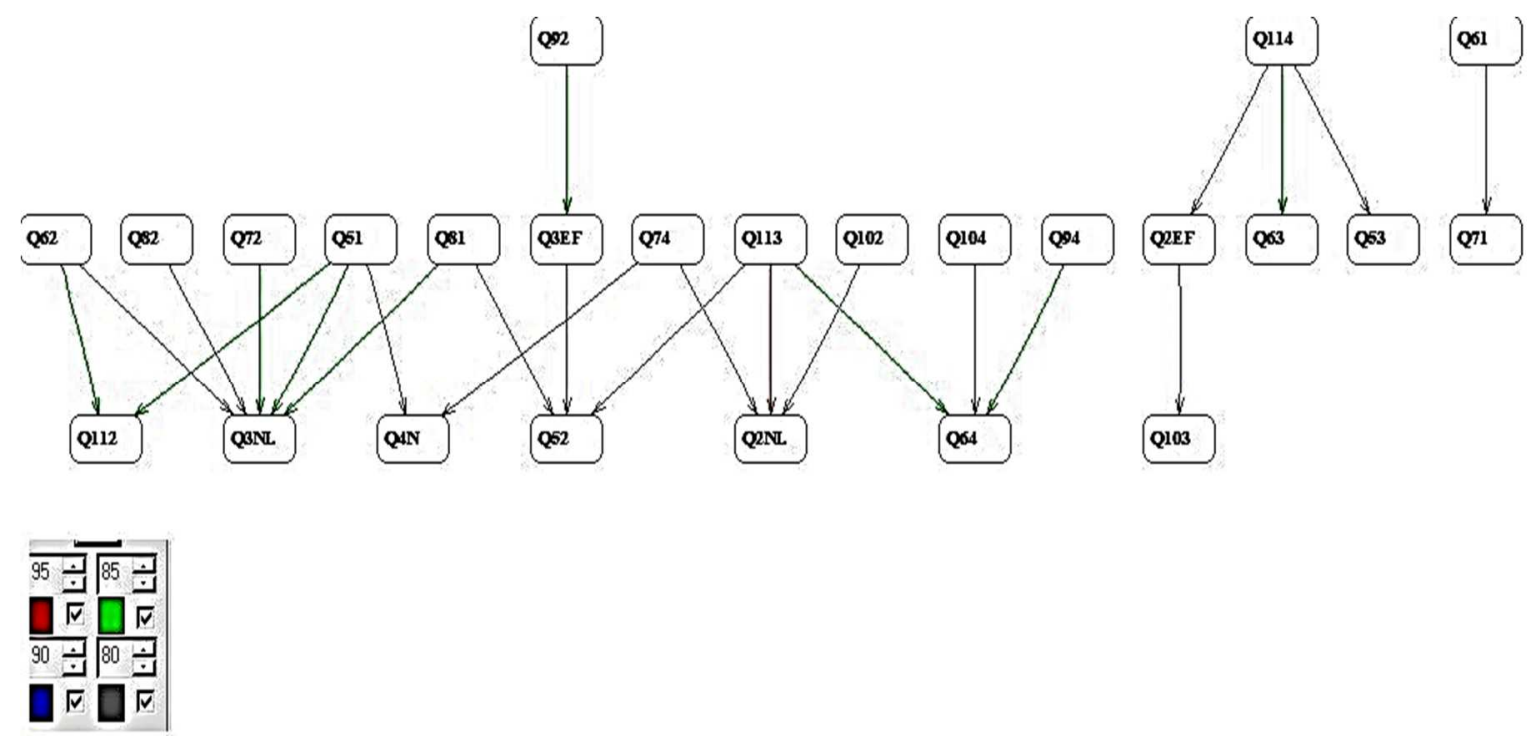

Fuente: El autor.

Leyenda de la figura 2:

Q113 - No recuerdo si estudiaste estadística en la escuela primaria.

Q2NL - Señaló la alternativa y en la pregunta 29 (alternativa incorrecta).

Q74 - Está totalmente de acuerdo en que las estadísticas son importantes para la toma de decisiones en su vida diaria.

Q102 - En parte no está de acuerdo con que la investigación estadística sea confiable.

Q3NL - No se acuerda si he estudiado estadística en la Secundaria.

Empezamos nuestro análisis con la ruta que tiene una flecha roja, es decir, con el mayor nivel de confianza: Q113 $\rightarrow$ Q2NL, lo que significa que se afirma saber cómo la investigación estadística es realizada, por lo que probablemente no recuerde si estudió estadística en escuela Primaria. La variable típica de este comportamiento son los hombres, con un riesgo de 0,195.

Dada la presencia masiva de estadísticas en los medios de comunicación, especialmente considerando el período electoral en el que se realizó la encuesta, podemos inferir que este 
grupo entendió la información proporcionada en esta investigación solo de manera intuitiva. Aquí hay un primer indicio de la necesidad de comenzar a abordar el contenido estadístico sin asumir un conocimiento estable.

Siguiendo los caminos que también terminan en Q2NL, pasamos a Q74 $\rightarrow$ Q2NL (cree que las estadísticas deberían usarse para la toma de decisiones diaria) y Q102 $\rightarrow$ Q2NL (no está de acuerdo con que la investigación estadística sea confiable). En ambos casos observamos las mismas variables típicas: sexo femenino con un riesgo de 0,246. Este grupo, a pesar de partir de premisas distintas, no recuerda si estudió estadística en la escuela primaria (Q2NL). Dichos caminos nos llevaron a reforzar la inferencia hecha en el párrafo anterior, sobre las necesidades para el inicio del acercamiento a la estadística con los estudiantes involucrados. Destacamos que, de los 86 entrevistados, 56 de ellos eligieron la opción que indicaba que no recordaban aprender estadística en la escuela primaria.

Otra variable también relacionada con varios caminos implicativos es el Q3NL, que significa no recordar haber estudiado estadística en la Secundaria. Recordamos que los entrevistados están en el tercer año de la escuela secundaria y aún no han cumplido con el contenido de estadística en el plan de estudios del Estado de São Paulo. 41 de los 86 encuestados indicaron esta opción.

De acuerdo con este plan de estudios, los estudiantes estudian probabilidad durante el segundo año de secundaria, lo que nos lleva a inferir que no asocian probabilidad con estadística. Cabe recordar que tanto en el Cuaderno del Estudiante (São Paulo, 2014) como en los libros de texto (Giordano, 2016) la estadística descriptiva se presenta de forma aislada. La siguiente tabla muestra las rutas encontradas.

Tabla 1. Caminos implicativos determinados en el análisis de las variables en juego.

\begin{tabular}{|l|l|}
\hline Caminos & Equivalencias \\
\hline Q62 $\rightarrow$ Q3NL & $\begin{array}{l}\text { No está de acuerdo que las estadísticas sean importantes para la comprensión de } \\
\text { las noticias en radio, televisión, Internet y periódicos. }\end{array}$ \\
\hline Q82 $\rightarrow$ Q3NL & $\begin{array}{l}\text { No está de acuerdo que se utilicen estadísticas en los cursos de educación superior } \\
\text { que pretende estudiar. }\end{array}$ \\
\hline Q72 $\rightarrow$ Q3NL & $\begin{array}{l}\text { No está de acuerdo con que la estadística es importante para la toma de decisión en } \\
\text { su vida cotidiana. }\end{array}$ \\
\hline Q51 $\rightarrow$ Q3NL & Afirma la falta de conocimiento acerca de la Estadística. \\
\hline Q81 $\rightarrow$ Q3NL & No cree plenamente que utilizará estadística en los cursos superiores. \\
\hline
\end{tabular}

Fuente: El autor. 
Los caminos marcados permitieron inferir la poca importancia que los estudiantes parecen atribuir a los conceptos estadísticos en su vida hasta ahora: no creen en su utilidad, en su vida diaria o en su futuro profesional, y afirman tener poco conocimiento estadístico sobre estos conceptos, lo que probablemente implica no recordar haber estudiado la asignatura en la secundaria. Estas inferencias se entienden a un nivel de confianza entre 0,85 y 0,90 . No diremos nada sobre las variables típicas, ya que el riesgo indicado en estas rutas es de aproximadamente 0,49, independientemente de la variable indicada. Todo eso nos lleva a inferir la necesidad de un abordaje cuidadoso del contenido estadístico, indicando la importancia del pleno desarrollo de la alfabetización estadística del estudiante, lo que ciertamente orienta las acciones de la fase experimental en la resolución de problemas luego del desarrollo de proyectos por parte de este.

A continuación, veremos un ejemplo de concepción movilizada por los estudiantes:

25 - (ENEM/2012) La siguiente tabla muestra la evolución de los ingresos brutos anuales en los últimos tres años de cinco microempresas (ME) que están a la venta.

Tabla 2. Evolución de los ingresos brutos anuales en los últimos tres años de cinco microempresas.

\begin{tabular}{|c|c|c|c|}
\hline ME & $\begin{array}{c}2009 \text { (em milhares } \\
\text { de reais) }\end{array}$ & $\begin{array}{c}2010 \text { (em milhares } \\
\text { de reais) }\end{array}$ & $\begin{array}{c}2011 \text { (em milhares } \\
\text { de reais) }\end{array}$ \\
\hline Alfinetes $V$ & 200 & 220 & 240 \\
\hline Balas $W$ & 200 & 230 & 200 \\
\hline Chocolates $X$ & 250 & 210 & 215 \\
\hline Pizzaria $Y$ & 230 & 230 & 230 \\
\hline Tecelagem $Z$ & 160 & 210 & 245 \\
\hline
\end{tabular}

Fuente: Datos ficticios

Un inversor quiere comprar dos de las empresas que figuran en la tabla. Para hacer esto, calcula el ingreso bruto anual promedio de los últimos tres años (de 2009 a 2011) y elige las dos compañías con el promedio anual más alto. Las empresas que este inversionista elige para hacer la comprar son:

a) Balas W y Pizzaria Y.

b) Chocolates X y Tecelagem Z.

c) Pizzaria $Y$ y Alfinetes V.

d) Pizzaria Y y Chocolates X.

e) Tecelagem $\mathrm{Z}$ y Alfinetes $\mathrm{V}$.

Pallauta, Gea y Venegas (2019) señalan que las tablas de distribución de frecuencias no reciben la debida atención en los planes de estudio y docencia, tanto en Chile como en Brasil, 
y agregan que estas tablas están prácticamente ausentes en los libros de texto de matemáticas en ambos países. Sin embargo, se encuentran en libros de otras disciplinas, como Geografía, lo que puede justificar que el $57 \%$ de los estudiantes respondan bien a la pregunta apuntando a la alternativa "d". Por otro lado, la opción equivocada con mayor número de opciones (19\% de estudiantes) fue la alternativa "e", que apunta a las dos empresas cuyos ingresos tuvieron la mayor amplitud total. Este argumento se puede reforzar con la redacción del enunciado de la pregunta, que menciona la "evolución" del ingreso bruto anual, lo que podría llevar al estudiante a pensar en la mayor variación entre los límites inferior y superior encontrados, respectivamente, en las empresas $\mathrm{Z}$ y $\mathrm{V}$.

Encontramos, detrás de esta respuesta, una posible concepción $\varnothing$, caracterizada por los elementos:

- P - campo del problema: determinación de la media aritmética simple a partir de los valores presentados en una tabla de doble entrada.

- R - operadores: ubicación de los valores de ingresos brutos anuales asociados a las respectivas empresas de origen en una tabla de distribución de frecuencias y comprobación de la diferencia entre ellas.

- L - conjunto de representaciones: representación numérica y representación tabular.

- $\sum$ - estructura de control: media aritmética simple como valor directamente proporcional a la variación entre los límites inferior y superior de la muestra (amplitud total).

Por tanto, podemos inferir la existencia de una posible concepción $\phi$, según la cual el valor medio será la diferencia más grande entre los dos extremos de la distribución de frecuencias: el límite inferior y el límite superior de la muestra. Esta idea podría ser considerada errónea por cualquier docente, pero la teoría ck $\not$ considera que las concepciones del sujeto que tienen una razón de existir, son intrínsecamente coherentes con su conocimiento de referencia, y deben tener un dominio de validez para él. Depende del profesor investigar, discutir con el estudiante y ofrecerle oportunidades para revisarlos y cambiarlos.

En cualquier trabajo estadístico cooperativo realizado en pequeños grupos, el choque de ideas, la discusión, la argumentación y la comprobación de hipótesis, como predice Garfield (1993, 2013), favorecen el esperado cambio en las concepciones y el refinamiento de la alfabetización, el razonamiento y el pensamiento estadístico. Díaz (2016) destaca que, según los estudiantes, el trabajo colaborativo en pequeños grupos reduce la ansiedad, lo que 
contribuye a una mejor comprensión de las nociones estadísticas y la adquisición de experiencias significativas.

Según este autor, las actividades colaborativas son más tranquilizadoras, motivadoras y estimulantes. La observación de los compañeros, trabajando activamente, favorece la concentración en la tarea y la aparición de una mayor diversidad de propuestas. El estímulo de la ayuda mutua permite la asimilación de conceptos, el avance de las actividades, la reducción de la percepción de dificultad de la tarea y la reducción de la ansiedad. El cuidado colectivo reduce la carga de las dificultades; intercambiar y compartir ideas mejora la autoconfianza y el compromiso colectivo (Díaz, 2016).

\section{Consideraciones Finales}

El abordaje estadístico a través de proyectos puede contribuir al cambio de concepciones de los estudiantes. Como se señaló en nuestra revisión de la literatura, hay pocos estudios publicados sobre concepciones desde la perspectiva del modelo ckф. Casi sin contacto previo con la estadística, en un entorno escolar, los estudiantes mostraron una comprensión y lectura mínima de tablas y gráficos estadísticos (niveles de lectura e interpretación gráfica uno y dos por Curcio (1987, 1989): leer los datos y leer entre datos), así como medidas de tendencia central y dispersión. Uno de los resultados de nuestro trabajo que consideramos más relevantes es que las estructuras de control manifestadas por un estudiante movilizan las estructuras cognitivas de otros, promoviendo la mejora de sus ideas y la revisión de sus propias concepciones, compartiendo el esquema de un gráfico estadístico con los colegas, revisando los pasos para calcular una medida de dispersión, comparando la determinación de una medida de tendencia central con otra obtenida por el colega, utilizando un método diferente y discutiendo su comprensión dentro del grupo. En relación a la variabilidad, los estudiantes reafirmaron o revisaron sus concepciones, mostrando cambios que pueden ser considerados como indicadores de aprendizaje, dentro del modelo ck $\varnothing$ de Balacheff $(1995,2002)$. El conocimiento del contexto, destacado por el modelo de alfabetización estadística de Gal (2019), tuvo un papel fundamental en la validación de las concepciones.

\section{Referencias}

Albani, P. (2015). Estatística com projetos: uma alternativa de ensino e aprendizagem. Disertación de Maestría. Universidad Tecnológica Federal do Paraná. 
Almouloud, S. A. (2007). Fundamentos da didática da matemática. Curitiba, Brasil: UFPR.

Almouloud, S. A.; Gras, R.; Régnier, J. A.S.I. (2014). Análise estatística implicativa: mais uma vez, o que é? Educação Matemática Pesquisa, São Paulo, 16 (3), 623-1087. Recuperado en 06 de septiembre de 2020, de https://revistas.pucsp.br/emp/article/view/21540/pdf_1

Balacheff, N. (1995). Conception, connaissance et concept. In: Grenier, D. (Ed.). Séminaire de l'équipe DidaTech. Grenoble: IMAG, p. 219-244. Recuperado en 06 de septiembre de 2020, de ouvertes.fr/file/index/docid/1072247/filename/1995_cKA_DDM95.PDF

https://hal.archives-

Balacheff, N. (2002). Cadre, registre et conception: note sur les relations entre trois concepts clés de la didactique. Les Cahiers du laboratoire Leibniz, 58, 1-18. Recuperado en 06 de septiembre de 2020, de http://pedagogie.lyon.iufm.fr/mathdelay/IMG/pdf/Balacheff.pdf

Balacheff, N. \& Gaudin, N. (2002). Student conceptions: An introduction to a formal characterization. Les Cahiers du Laboratoire Leibnitz 65, p.1-21. Recuperado en 06 de septiembre de 2020, de https://telearn.archives-ouvertes.fr/hal-00190425/document

Barberino, M. R. B. (2016). Ensino de estatística através de projetos. Dissertação de Mestrado. Universidade de São Paulo, Brasil.

Batanero, C. (1999). Taller sobre análisis exploratorio de datos en la enseñanza secundaria. In Actas de la Conferencia Internacional "Experiencias e Expectativas do Ensino de Estatística: Desafios para o Século XXI'. Florianópolis, Santa Catarina, Brasil. Recuperado en 06 de septiembre de 2020, de https://www.researchgate.net/profile/Carmen_Batanero/publication/282281205 Taller_sob re analisis_exploratorio_de_datos_en_la ensenanza_secundaria/links/560ce08c08aea6865 3d3868f.pdf

Batanero, C. (2001). Didáctica de la Estadística. Granada: Departamento de Didáctica de la Matemática de la Universidad de Granada.

Batanero, C. \& Díaz, C. (2004) El papel de los proyectos en la enseñanza y aprendizaje de la estadística. Em J. P. Royo (Ed.). Aspectos didácticos de las matemáticas (pp. 125164). Zaragoza: ICE.

Batanero, C. \& Díaz, C. (2011). Estadística con proyectos. Granada: Universidad de Granada.

Batanero, C., Estepa, A. \& Godino, J. D. (1991). Análisis exploratorio de datos: sus posibilidades en la enseñanza secundaria. Suma, 9, 25-31. Recuperado en 06 de septiembre de 2020, de http://www.cvrecursosdidacticos.com/web/repository/1293458560_Analisis exploratorio SUMA 91.pdf

Batanero, C., Godino, J., Green, D., Holmes, P., \& Vallecillos, A. (1994). Errores y dificultades en la comprensión de los conceptos estadísticos elementales. International Journal of Mathematics Education in Science and Technology, 25(4), 527-547. Recuperado en 06 de septiembre de 2020, de https://www.researchgate.net/profile/Carmen Batanero/publication/237768038 ERRORE S Y DIFICULTADES EN LA COMPRENSION DE LOS CONCEPTOS ESTADIS TICOS ELEMENTALES/links/0046352222434f269a000000.pdf

Brasil. (2018). Base Nacional Comum Curricular - Educação é a Base: Ensino Médio. Brasília: Ministério da Educação e Cultura, Brasília. 
Cobello, L. S. (2018) Letramento Estatístico: análise e reflexões sobre as tarefas contidas no material didático da secretária estadual de educação de São Paulo para o Ensino Médio. Dissertação de Mestrado. São Carlos: Universidade Federal de São Carlos. UFSCar.

Conti, K. C. (2009). O papel da estatística na inclusão de alunos da educação de jovens e adultos em atividades letradas. Dissertação de Mestrado. UNICAMP.

Costa, G. D. F. D. (2012). A metodologia de projetos como uma alternativa para ensinar estatística no ensino superior. Tese de Doutorado. UNICAMP.

Coutinho, C. Q. S., \& Miguel, M. I. R. (2007). Análise Exploratória de Dados: um estudo diagnóstico sobre concepções de professores. 30 Reunião Anual da ANPED. Anais da $30^{a}$ Reunião Anual da ANPED, Caxambu-MG. Recuperado en 06 de septiembre de 2020, de https://anped.org.br/reunioes-cientificas/nacional

Couturier, R., Bodin, A., \& Gras, R. (2004). A classificação hierárquica implicativa e coesiva. Manual Curso CHIC versão, 2. Recuperado en 06 de septiembre de 2020, de http://math.unipa.it/ grim/asi/asi_03_gras_bodin_cout.pdf

Couturier, R. D. \& Gras, R. (2005). CHIC: traitement de données avec l'analyse implicative. En C. Ritschard y Djeraba (Eds.), Journées d'extraction et gestion des connaissances (EGC'2005) (Vol.2, pp. 679-684). Recuperado en 06 de septiembre de 2020, de https://www.researchgate.net/profile/Regis_Gras/publication/220786956_CHIC traitemen $\mathrm{t}$ de donnees_avec l'analyse implicative/links/5c18c2ab92851c39ebf56b52/CHICtraitement-de-donnees-avec-lanalyse-implicative.pdf

Creswell, J. W. (2010). Projeto de pesquisa métodos qualitativo, quantitativo e misto. Porto Alegre: Artmed.

Curcio, F. R. (1987). Comprehension of mathematical relationships expressed in graphs. Journal for research in mathematics education, 382-393. Recuperado en 06 de septiembre de 2020, de https://www.jstor.org/stable/749086

Curcio, F. R. (1989). Developing Graph Comprehension. Elementary and Middle School Activities. National Council of Teachers of Mathematics, Inc., 1906 Association Drive, Reston, VA 22091. Recuperado el 06 de septiembre de 2020, de https://eric.ed.gov/?id=ED314245

Dangió, E. G. Z. (2014). O ensino de estatística no Ensino Médio através de projetos. Dissertação de Mestrado, Universidade Federal de São Carlos, São Carlos.

Diaz, D. (2016). Les facteurs influençant la réussite des activités collaboratives médiées par les TICE dans une situation de formation universitaire à la statistique. (Doctoral dissertation, Thèse de doctorat (Dirigée par Jean-Claude Régnier) Lyon 2, Lyon, France, 2016.

Fischbein, E. (1982). Intuition and proof. For the learning of mathematics, 3(2), 9-24. Recuperado en 06 de septiembre de 2020, de https://pdfs.semanticscholar.org/9ee2/b4dd3ab754cceef68d14e4950584deaf8a45.pdf 
Freitas, M. M. (2016) Praticando Estatística no Ensino Médio. Dissertação de Mestrado. Universidade de Brasília, DF, 2016.

Gal, I. (2019) Understanding statistical literacy: About knowledge of contexts and models. En J. M. Contreras, M. M. Gea, M. M. López-Martín y E. Molina-Portillo (Eds.). Actas del Tercer Congreso Internacional Virtual de Educación Estadística. Granada, España. Recuperado el 06 de septiembre de 2020, de http://www.ugr.es/ fqm126/civeest.html

Garfield, J. (1993) Teaching statistics using small-group cooperative learning. Journal of Statistics Education, v. 1, n. 1, p. 1-9. Recuperado en 06 de septiembre de 2020, de https:/www.tandfonline.com/doi/full/10.1080/10691898.1993.11910455?scroll=top\&need Access $=$ true\&utm_source $=$ TrendMD\&utm medium $=$ cpc\&utm campaign $=$ Journal_of_Sta tistics Education TrendMD 0

Garfield, J. (2013) Cooperative learning revisited: From an instructional method to a way of life. Journal of Statistics Education, v. 21, n. 2, p. 1-8. Recuperado en 06 de septiembre de 2020, de https://www.tandfonline.com/doi/pdf/10.1080/10691898.2013.11889673

Giordano, C. C. (2016). O desenvolvimento do letramento estatístico por meio de projetos: um estudo com alunos do ensino médio. Dissertação de Mestrado. Pontifícia Universidade Católica de São Paulo.

Giordano, C. C. (2020). Concepções estatísticas: um estudo com alunos do Ensino Médio. Tese de Doutorado. Pontifícia Universidade Católica de São Paulo.

Gras, R. (2015). O Uso do CHIC na Formação de Educadores. JA Valente, \& MEB Almeida. Uso do CHIC na Formação de Educadores: à guisa de apresentação dos fundamentos e das pesquisas e foco. Rio de Janeiro: Letra Capital.

Gras, R., \& Almouloud, S. A. (2002). A implicação estatística usada como ferramenta em um exemplo de análise de dados multidimensionais. Educ Mat Pesqui, 4(2), 75-88. Recuperado en 06 de septiembre de 2020 , de http://dipmat.math.unipa.it/ grim/asi/asi_03_saddo_gras.pdf

Gras, R., Régnier, J. C.; Marinica, C. \& Guillet, F. (2013) L'analyse statistique implicative Méthode exploratoire et confirmatoire à la recherche de causalités. Toulouse: Cépaduès Editions.

Melo, K. M. F. (2017) O pensamento estatístico no ensino fundamental: uma experiência articulando o desenvolvimento de projetos de pesquisa com os conceitos básicos da estatística implementados em uma sequência didática eletrônica. Tese de Doutorado. Universidade Luterana do Brasil.

Mendonça, L. D. O. (2008) A Educação Estatística em um ambiente de modelagem matemática no ensino médio. Dissertação de Mestrado. São Paulo: Universidade Cruzeiro do Sul.

Oliveira, A. F. D. (2019). Práticas pedagógicas no Ensino Médio: por uma estatística crítica e contextualizada. Dissertação de Mestrado. Universidade Federal de Goiás. Goiânia. 
Oliveira, P. G. D. (2010). Probabilidade: concepções construídas e mobilizadas por alunos do Ensino Médio à luz da teoria das concepções $(\mathrm{CK} \phi)$. Dissertação de Mestrado. Pontifícia Universidade Católica de São Paulo, São Paulo.

Oliveira, P. G. D., \& Coutinho, C. D. Q. (2011). Concepções probabilísticas à luz da Teoria $\mathrm{CK} \phi(\mathrm{CO})$. In Anais da XIII Conferência Interamericana de Educação Matemática. Recife: UFPE. Recuperado en 06 de septiembre de 2020, de http://xiii.ciaemredumate.org/index.php/xiii_ciaem/xiii_ciaem/paper/view/578

Orús, P., Zamora, L., \& Gregori, P. (2009). Teoría y aplicaciones del Análisis Estadístico Implicativo: primera aproximación en lengua hispana. Castelló de la Plana: Innovació Digital Castelló

Pallauta, J. D.; Gea, M. M. S.; Venegas, A. G. (2019) Las actividades sobre tablas estadísticas en textos escolares chilenos de educación básica. In: Congreso Internacional Virtual de Educación Estadística. Granada. Anais. Recuperado en 06 de septiembre de 2020, de https://digibug.ugr.es/bitstream/handle/10481/55209/pallauta.pdf?sequence=1\&isAllowed $=\mathrm{y}$

Porciúncula, M. \& Samá, S. (2015). Projetos de aprendizagem: uma proposta pedagógica para a sala de aula de estatística. Em M. Porciúncula e S. Samá (Eds.), Educação Estatística: ações e estratégias pedagógicas no Ensino Básico e Superior. Curitiba: Editora CRV.

Prado, M. (2002). O uso do CHIC na análise de registros textuais em ambiente virtual de formação de professores. Revista do Programa de Pós-Graduados em Educação Matemática - PUC-SP 4(2), 103-123. Recuperado el 06 de septiembre de 2020, de file://C:/Users/ccgio/Downloads/560-317-PB.pdf

Quirós, M. M. V. (2012). El aprendizaje estadístico en la Educación Secundaria Obligatoria a través de una metodología por proyectos: estudio de caso en un aula inclusiva. Tesis doctoral. Granada: Universidad de Granada.

Santana, M. D. S. (2011). A Educação Estatística com base num ciclo investigativo: um estudo do desenvolvimento do letramento estatístico de estudantes de uma turma do $3^{\circ}$ ano do Ensino Médio. Dissertação de Mestrado - Universidade Federal de Ouro Preto, Ouro Preto.

São Paulo (2012). Currículo do estado de São Paulo - Matemática e suas tecnologias: Ensino Fundamental Ciclo II e Ensino Médio. SE/CENP, São Paulo.

São Paulo (2014) Proposta curricular: caderno do aluno - Ensino Médio: Matemática, v. 2. IMESP, São Paulo.

Tukey, J. W. (1970). Exploratory data analysis. Reading: Addison-Wesley.

Vadcard, L. (2000). Etude de la notion d'angle sous le point de vue des conceptions (Doctoral dissertation, Grenoble 1). 


\section{Apéndice I - Cuestionario}

1 - ¿Qué son las estadísticas? ¿Es una ciencia exacta o una ciencia social? ¿Es un área de matemáticas?

2 - ¿Has estudiado Estadística en la escuela primaria? ¿Qué año?

( ) $1^{\circ}$ ( ) $2^{\circ}$ ( ) $3^{\circ}$ ( ) $4^{\circ}$ ( ) $5^{\circ}$ ( ) $6^{\circ}$ ( ) $7^{\circ}$ ( ) $8^{\circ}$ ( ) $9^{\circ}$ ( ) no recuerda ( ) nunca estudió 3 - ¿Has estudiado estadística en la secundaria? ¿Qué año?

( ) $1^{\circ}$ ( ) $2^{\circ}$ ( ) $3^{\circ}$ ( ) $9^{\circ}$ ( ) no recuerda ( ) nunca estudió

4 - ¿Has estudiado Estadística en otro curso, fuera de la educación regular?

( ) No ( ) Sí, estudié en el curso (s):

5 - Califica tu nivel de conocimiento en Estadística.

$\begin{array}{llll}1 & 2 & 3 & 4\end{array}$

Falta de conocimiento $\mathbb{C} \quad \mathbb{C} \quad \mathbb{C} \quad \mathbb{C}$ Dominio perfecto

6 - ¿Es importante la estadística para entender las noticias de la radio, la televisión, internet, los periódicos?

$\begin{array}{llll}1 & 2 & 3 & 4\end{array}$

Muy en desacuerdo $\mathbf{C} \quad \mathbb{C} \quad \mathbb{C} \quad \mathbb{C}$ Totalmente de acuerdo

7 - ¿Estás de acuerdo en que las estadísticas son importantes para tomar decisiones en tu vida diaria?

$\begin{array}{llll}1 & 2 & 3 & 4\end{array}$

Muy en desacuerdo $\mathbb{C} \quad \mathbb{C} \quad \mathbb{C} \quad \mathbb{C} \quad$ Totalmente de acuerdo

8 - ¿Crees que usarás Estadísticas en los cursos superiores que pretendes estudiar?

$\begin{array}{llll}1 & 2 & 3 & 4\end{array}$

No creo $\mathbb{C} \quad \mathbb{C} \quad \mathbb{C}$ Creo totalmente

9 - ¿Crees que la estadística es un área de las matemáticas, que lleva una ciencia exacta?

$\begin{array}{llll}1 & 2 & 3 & 4\end{array}$

No creo $\square \square \square \quad \square \quad C \quad$ Creo totalmente

$\mathbf{1 0}$ - ¿Confías en la investigación estadística?

$$
\begin{array}{llll}
1 & 2 & 3 & 4
\end{array}
$$

No confío $\mathbb{C} \quad \mathbb{C} \quad \mathbb{C} \quad \mathbb{C} \quad$ Confío totalmente

11 - ¿Sabes cómo se lleva a cabo la investigación estadística?

$$
\begin{array}{llll}
1 & 2 & 3 & 4
\end{array}
$$

No sé $\square \square \square \square \quad C \quad$ Yo sé muy bien 
12 - ¿Has estudiado Probabilidad en la escuela primaria? ¿Qué año?
( ) $1^{\circ} \quad\left(\right.$ ) $2^{\circ}$
( ) $3^{\circ}$ ( ) $4^{\circ}$ ( ) $5^{\circ}$
( ) $6^{\circ}$
( ) $7^{\circ}$
( ) $8^{\circ}$ ( ) $9^{\circ}$ ( ) no recuerda
( ) nunca estudió

13 - ¿Has estudiado Probabilidad en la escuela secundaria? ¿Qué año?

( ) $1^{\circ}$ ( ) $2^{\circ}$ ( ) $3^{\circ}$ ( ) no recuerda ( ) nunca estudió

14 - ¿Has estudiado Probabilidad en otro curso, fuera de la educación regular?

( ) No ( ) Sí, estudié en el curso (s):

15 - Califique su nivel de conocimiento de probabilidad:

$$
\begin{array}{llll}
1 & 2 & 3 & 4
\end{array}
$$

Falta de conocimiento $\mathbb{C} \quad \boldsymbol{C} \quad \mathbb{C} \quad \mathbb{C}$ Dominio perfecto

En las siguientes declaraciones, indique su nivel de acuerdo, siguiendo la escalera a continuación:

1. totalmente en desacuerdo;

2- parcialmente en desacuerdo;

3- parcialmente de acuerdo;

4- muy de acuerdo.

16 - En un juego de dos dados, numerados del 1 al 6, ¿Cuál son las posibilidades de obtener la suma de los dos igual a 10 ?

17 - En un sorteo de Mega-Sena, las posibilidades de que alguien gane con la apuesta: 1, 2, 3, 4, 5, 6 son menores que las posibilidades de ganar con la apuesta: $5,12,23,38,45,56$ ?

18 - En el caso anterior, esta sería una buena apuesta, ya que, siendo un resultado inusual, si alguien ganara, compartiría el premio con un número menor de apostadores, ¿estaría de acuerdo?

19 - ¿No cae un rayo dos veces en el mismo lugar?

20 - ¿La posibilidad de que una persona muera cuando salta desde el piso 12 de un edificio directamente al asfalto, es el doble de posibilidades de morir si salta desde el piso 6 ?

21 - Cuando nuestra fila en la caja de un supermercado o en un peaje de carretera es muy lenta, ¿es siempre una buena opción cambiar a la siguiente línea, que es más rápida?

22 - Barajé las cartas de un mazo tradicional y extraje aleatoriamente una jota de corazones, volviéndola a colocar en la pila. En un segundo sorteo, ¿la posibilidad de obtener otra jota de corazones es menor que la de sacar otras cartas?

23 - El sábado, en el pronóstico del tiempo, se anunció que la probabilidad de que no lloviera en la ciudad de São Paulo al día siguiente sería de 90\%. Sin embargo, llovió el domingo. ¿Estaba mal el pronóstico del tiempo?

24 - Valquíria quiere firmar su coche con las iniciales de su nombre y los días de cumpleaños de ella (22) y su hija (13), pero se enteró de que la placa de matrícula VAL2213 es rara, esta opción es más difícil que la mayoría de las otras. ¿Estás de acuerdo con esta información?

25 - (ENEM/2012) La siguiente tabla muestra la evolución de los ingresos brutos anuales en los últimos tres años de cinco microempresas $(\mathrm{ME})$ que están a la venta.

\begin{tabular}{|c|c|c|c|}
\hline ME & $\begin{array}{c}2009 \text { (em milhares } \\
\text { de reais) }\end{array}$ & $\begin{array}{c}\mathbf{2 0 1 0} \text { (em milhares } \\
\text { de reais) }\end{array}$ & $\begin{array}{c}\text { 2011 (em milhares } \\
\text { de reais) }\end{array}$ \\
\hline Alfinetes $V$ & 200 & 220 & 240 \\
\hline Balas $W$ & 200 & 230 & 200 \\
\hline Chocolates $X$ & 250 & 210 & 215 \\
\hline Pizzaria $Y$ & 230 & 230 & 230 \\
\hline Tecelagem $Z$ & 160 & 210 & 245 \\
\hline
\end{tabular}

Fuente: datos ficticios 
Un inversor quiere comprar dos de las empresas que figuran en la tabla. Para hacer esto, calcula el ingreso bruto anual promedio de los últimos tres años (de 2009 a 2011) y elige las dos compañías con el promedio anual más alto. Las empresas que este inversionista elige comprar son:

a) Balas W y Pizzaria Y.

b) Chocolates $X$ y Tecelagem $Z$.

c) Pizzaria Y y Alfinetes V.

d) Pizzaria Y y Chocolates X.

e) Tecelagem $\mathrm{Z}$ y Alfinetes $\mathrm{V}$.

26 - (ENEM/2010) La siguiente tabla muestra el desempeño de un equipo de fútbol en la última liga. La columna de la izquierda muestra la cantidad de goles marcados y la columna de la derecha indica cuántos juegos marcó el equipo esa cantidad de goles.

\begin{tabular}{|c|c|}
\hline Gols marcados & Quantidade de partidas \\
\hline 0 & 5 \\
\hline 1 & 3 \\
\hline 2 & 4 \\
\hline 3 & 3 \\
\hline 4 & 2 \\
\hline 5 & 2 \\
\hline 7 & 1 \\
\hline
\end{tabular}

Fuente: datos ficticios

Si X, Y y Z son, respectivamente, la media, la mediana y la moda de esta distribución, entonces:
a) $\mathrm{X}=\mathrm{Y}<\mathrm{Z}$.
b) $\mathrm{Z}<\mathrm{X}=\mathrm{Y}$.
c) $\mathrm{Y}<\mathrm{Z}<\mathrm{X}$.
d) $\mathrm{Z}<\mathrm{X}<\mathrm{Y}$.
e) $\mathrm{Z}<\mathrm{Y}<\mathrm{X}$.

27 - (ENEM/2012) El gráfico muestra el comportamiento del trabajo formal que surgió, según CAGED, de enero de 2010 a octubre de 2010.

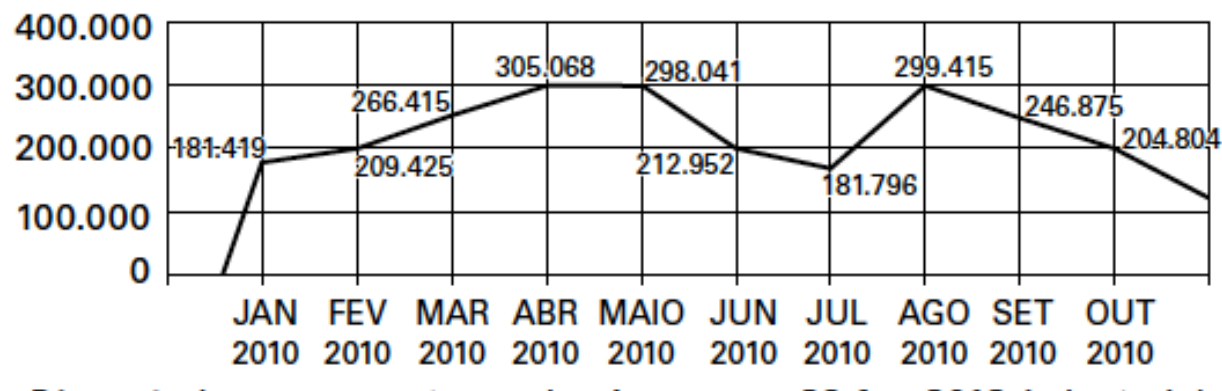

Disponivel em: www.mte.gov.br. Acesso em: 28 fev. 2012 (adaptado).

Según el gráfico, el valor de la porción mediana completa de los trabajos formales en el período es:
a) 212.952 .
b) 229.913 .
c) 240.621 .
d) 255.496 .
e) 298.041 .

28 - (ENEM/2010) Marco y Paulo fueron clasificados en un concurso. Para la clasificación en la competencia, el candidato debe obtener un promedio aritmético en el puntaje igual o mayor a 14. En 
caso de empate en el promedio, el desempate estaría a favor del puntaje más regular. La siguiente tabla muestra los puntos obtenidos en las pruebas de Matemáticas, Portugués y Conocimientos Generales, la media, la mediana y la desviación estándar de los dos candidatos.

Detalles de los candidatos en la competencia.

\begin{tabular}{|l|c|c|c|c|c|c|}
\hline & Matemática & Português & $\begin{array}{c}\text { Conhecimentos } \\
\text { Gerais }\end{array}$ & Média & Mediana & $\begin{array}{c}\text { Desvio } \\
\text { Padrão }\end{array}$ \\
\hline Marco & 14 & 15 & 16 & 15 & 15 & 0,32 \\
\hline Paulo & 8 & 19 & 18 & 15 & 18 & 4,97 \\
\hline
\end{tabular}

Fuente: datos ficticios

El candidato con el puntaje más regular, por lo tanto, el más alto en la competencia, es:

a) Marco, ya que la media y la mediana son iguales.

b) Marco, ya que obtuvo menos desviación estándar.

c) Paulo, porque obtuvo el puntaje más alto en la tabla, 19 en portugués.

d) Paulo, ya que obtuvo la mediana más alta.

e) Paulo, ya que obtuvo una mayor desviación estándar.

29 - (ENEM/2010) Los datos del gráfico siguiente fueron generados a partir de datos recogidos en el conjunto de seis regiones metropolitanas por el Dieese.

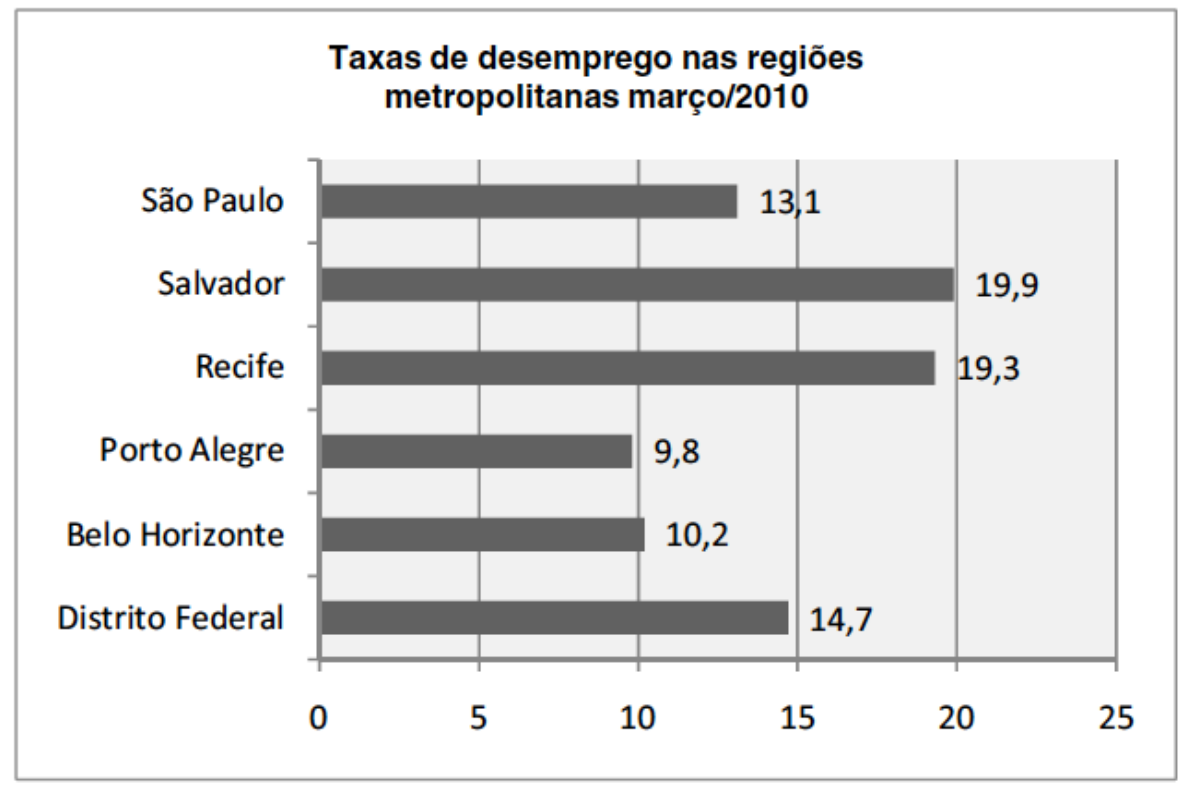

Disponível em: http://g1.globo.com. Acesso em: 28 abr. 2010 (adaptado).

Suponiendo que el número total de personas encuestadas en la región metropolitana de Porto Alegre es equivalente a 250,000, el número de desempleados en marzo de 2010, en esa región, fue:
a) 24500 .
b) 25000 .
c) 220500 .
d) 223000 .
e) 227500 . 


\section{Apéndice II - Actividad en grupos}

En una escuela pública en Santo André, un grupo de estudiantes de secundaria de tercer año, que realizaban una encuesta escolar, decidió investigar la opinión de sus colegas sobre la política de cuotas de admisión a las universidades federales (públicas). Esta escuela tenía 938 estudiantes, 417 estudiantes de primaria, estudiando en la tarde (6to a 9no grado) y 521 en la secundaria, estudiando en la mañana (204 en 1er grado, 179 en 2do grado y 138 en 3 er grado). De estos 938 estudiantes, 513 son mujeres. Este grupo decidió entrevistar a 40 estudiantes, 20 (diez hombres y diez mujeres) del período de la mañana y 20 (diez hombres y diez mujeres) del período de la tarde. Preparó un cuestionario con doce preguntas, cuyas respuestas se pueden observar en la base de datos que sigue. Analice los resultados utilizando las representaciones que considere apropiadas y las medidas que considere apropiadas. Recuerde siempre de explicar su pensamiento como sea posible.

a) Con respecto a la variable "edad", los estudiantes proporcionaron las siguientes respuestas:

\begin{tabular}{|l|l|l|l|l|l|l|l|l|l|l|l|l|}
\hline Edad (años) & 11 & 12 & 13 & 14 & 15 & 16 & 17 & 18 & 19 & 20 & 21 & Total \\
\hline Frecuencia simple & 3 & 4 & 4 & 6 & 5 & 8 & 4 & 3 & 2 & 0 & 1 & 40 \\
\hline
\end{tabular}

¿Cómo explica la variación de edades usando las herramientas estadísticas que ha aprendido? Construya una o más representaciones gráficas, explicándolas. Determine una o más medidas de resumen (media, moda, mediana, cuartiles, desviación estándar, amplitud) y explique su significado cuando queremos analizar este conjunto de edades en el grupo de 40 estudiantes encuestados.

b) Con respecto al ingreso familiar, los estudiantes obtuvieron las siguientes respuestas

\begin{tabular}{|l|l|l|l|l|l|l|l|}
\hline Sujeto & $\begin{array}{c}\text { Ingreso } \\
\text { Familiar (R\$) }\end{array}$ & Sujeto & $\begin{array}{c}\text { Ingreso } \\
\text { Familiar } \\
(\mathrm{R} \$)\end{array}$ & Sujeto & $\begin{array}{c}\text { Ingreso } \\
\text { Familiar (R\$) }\end{array}$ & Sujeto & $\begin{array}{c}\text { Ingreso } \\
\text { Familiar(R\$) }\end{array}$ \\
\hline 1 & 1108,38 & 11 & 4800 & 21 & 3000 & 31 & No sepa \\
\hline 2 & 1500 & 12 & 5100 & 22 & 1108,38 & 32 & 3328,14 \\
\hline 3 & 2521 & 13 & 1108,38 & 23 & No declarado & 33 & 4623 \\
\hline 4 & No declarado & 14 & 1108,38 & 24 & 3820 & 34 & 4100 \\
\hline 5 & 2800 & 15 & 1500 & 25 & 2216,76 & 35 & 5000 \\
\hline 6 & 1108,38 & 16 & No sepa & 26 & 2600 & 36 & 2400 \\
\hline 7 & 3500 & 17 & 2750 & 27 & 1200 & 37 & 1108,38 \\
\hline 8 & 4714 & 18 & 3400 & 28 & 1108,38 & 38 & 1700 \\
\hline 9 & 1108,38 & 19 & No declarado & 29 & 30000 & 39 & 6330 \\
\hline 10 & 2200 & 20 & 1108,38 & 30 & 1300 & 40 & No sepa \\
\hline
\end{tabular}

¿Cómo explica la variación en los ingresos declarados usando las herramientas estadísticas que ha aprendido? Construya una o más representaciones gráficas, explicándolas. Determine una o más medidas de resumen (media, moda, mediana, cuartiles, desviación estándar, amplitud) y explique su significado cuando queramos analizar este conjunto de ingresos familiares en el grupo de 40 estudiantes encuestados.

c) Con respecto a la educación de los padres, los estudiantes prepararon la siguiente tabla. La tabla hecha simplifica la base de datos. ¿Cómo evidenciaría esta comparación si quisiera usar gráficos? Explique su razonamiento. ¿Es posible determinar una medida de resumen que contribuya al análisis de estos datos? ¿Cual? ¿Cómo determinarlo? Explique su razonamiento. 


\begin{tabular}{|l|c|c|}
\hline Escolaridad & Del padre & De la madre \\
\hline No alfabetizado & 2 & 1 \\
\hline Primaria incompleta & 2 & 1 \\
\hline Primaria completa & 4 & 6 \\
\hline Secundaria incompleta & 4 & 0 \\
\hline Secundaria completa & 9 & 15 \\
\hline Pregrado incompleto & 6 & 1 \\
\hline Pregrado completo & 8 & 12 \\
\hline Posgrado incompleto & 3 & 0 \\
\hline Posgrado completo & 2 & 4 \\
\hline Total & 40 & 40 \\
\hline
\end{tabular}

Autor

Cassio Cristiano Giordano, Faculdades Integradas de Guarulhos (FG-SP) Doutor em Educação Matemática pela Pontifícia Universidade Católica de São Paulo, com estágio doutoral na Université de Franche-Comté - Besançon/França. Mestre em Educação Matemática pela Pontifícia Universidade Católica de São Paulo. Especialista em Ensino da Matemática, pela Universidade Estadual de Campinas; em Novas Tecnologias no Ensino da Matemática, pela Universidade Federal Fluminense; em Docência e Pesquisa no Ensino Superior, pela Universidade Metropolitana de Santos; em Matemática no Ensino Médio, pela Pontifícia Universidade Católica de São Paulo. Licenciado em Ciências e Matemática pela Universidade Ibirapuera. Licenciado em Pedagogia pela Universidade Metropolitana de Santos. Bacharel em Psicologia pela Universidade Metodista de São Paulo. 